\title{
Direct Measurements of Thermal Vacancies in $\mathrm{BCC}{ }^{4} \mathrm{He}$
}

\author{
P. R. Granfors, ${ }^{*}$ B. A. Fraass, $\dagger$ and R. O. Simmons \\ Department of Physics and Materials Research Laboratory, University of Illinois \\ at Urbana-Champaign, Urbana, Illinois
}

(Received November 14, 1986)

\begin{abstract}
$X$-ray lattice parameters of bcc ${ }^{4} \mathrm{He}$ crystals in contact with superfluid ${ }^{4} \mathrm{He}$ were measured with $300 \mathrm{ppm}$ accuracy in the temperature range $1.494-1.711 \mathrm{~K}$, corresponding to solid molar volumes between 20.88 and $21.09 \mathrm{~cm}^{3}$. Thermal vacancy concentrations in the solid at melting were estimated from a comparison of these lattice parameters with bulk solid molar volumes from the literature. Independently, temperature-dependent $x$-ray lattice parameter measurements on a bcc sample, held at constant macroscopic volume from 1.700 to $1.735 \mathrm{~K}$, were referenced to the same solid sample (at $20.90 \mathrm{~cm}^{3}$ ) in the hcp phase near $0.8 \mathrm{~K}$, where thermal vacancy content is small. The value of the free energy of formation for vacancies in bcc ${ }^{4} \mathrm{He}$ obtained from these measurements is $\mathrm{f}=9 \pm 1 \mathrm{~K}$. The relationship between this value and indirect inferences about vacancy formation from ion mobility and NMR experiments is discussed. Comparisons are made between vacancy properties in bcc ${ }^{4} \mathrm{He}$ and in the more extensively studied bcc phase of ${ }^{3} \mathrm{He}$. There are many parallels between the two systems. But a puzzling apparent difference between the vacancy relaxation energies in the two solids is pointed out.
\end{abstract}

\section{INTRODUCTION}

At least three types of excitations are important in the solid heliums ${ }^{4} \mathrm{He}$ and ${ }^{3} \mathrm{He}$; phonons, excitations associated with the exchanges of atoms, and vacancies. First, effects on thermodynamic properties of the solid due to phonons, especially at small molar volumes, are similar to phonon effects in classical solids, even though large renormalizations of the phonon frequencies occur due to the nonlocalization of atoms about atomic sites.

*Present address: General Electric Co., Milwaukee, Wisconsin.

$\dagger$ Present address: Department of Radiation Therapy, University of Michigan Hospitals, Ann Arbor, Michigạn. 
Deviations appear as interference effects between one-phonon and multiphonon excitation in experiments using the scattering of light and of neutrons. ${ }^{1}$ Second, atomic exchange effects arise, even at the lowest temperatures, from the extreme nonlocalization of atoms. ${ }^{2}$ Such exchange and its consequences have been examined most extensively for the magnetic properties of ${ }^{3} \mathrm{He}$, both experimentally and theoretically. ${ }^{3}$ Third, at the highest temperatures in solid ${ }^{3} \mathrm{He}$, thermal vacancy excitations have been directly identified by $x$-ray diffraction. ${ }^{4,6}$ Calculations and speculations, especially about vacancies in ${ }^{3} \mathrm{He}$ and ${ }^{3} \mathrm{He}-{ }^{4} \mathrm{He}$ mixtures, have been numerous and have been reviewed extensively. ${ }^{3,7-9}$ However, vacancies in pure bcc ${ }^{4} \mathrm{He}$ have rarely been studied. ${ }^{10,11}$ We report here the first direct measurements of thermal vacancy concentrations in bcc ${ }^{4} \mathrm{He}$. This phase exists over only a very narrow range of temperature, pressure, and molar volume $^{12,13}$ (Fig. 1).

The most direct, if not the most sensitive, way to obtain information about vacancies in solids, and the method used in the present study, is through comparison of densities. The method is based on the fact that the net added concentration $c$ of substitutional atomic sites in a crystal at a given temperature is proportional to the difference between the bulk volume $V$ and the volume as determined by $\mathrm{x}$-ray diffraction $V_{\mathrm{x}}\left(V_{\mathrm{x}}=a^{3} / 2\right.$ for bcc crystals, if $a$ is the cubic lattice parameter):

$$
c=\left(V-V_{\mathrm{x}}\right) / V_{\mathrm{x}}
$$

Equation (1) has this simplicity because the volume changes arising from the dilatational fields of point defects make equal contributions to the macroscopic volume change and to the volume change as measured by the coherent Bragg scattering. Accurate theoretical analysis of this question began with Eshelby, ${ }^{14}$ who used an elastic continuum approach, and there have been many subsequent treatments of various aspects of the problem: crystal and defect anisotropy, lattice anharmonicity, dense defect concentrations, etc. We are not aware, however, of any rigorous comprehensive theoretical justification that Eq. (1) remains valid when all such aspects are present simultaneously. In this absence, perhaps the best justification is empirical. A strong example is the excellent agreement between simultaneous bulk and $\mathrm{x}$-ray diffraction measurements, over a very wide range, of the thermal expansion of $\mathrm{AgCl},{ }^{15}$ known to contain dense concentrations of thermal Frenkel defects. Such defects do produce significant dilatational fields, yet their formation does not produce additional atomic sites.

Another aspect of Eq. (1) not deeply probed either theoretically or empirically is the question of its validity for nonlocalized defects, which are supposed to exist in solid helium under certain conditions. ${ }^{7-9}$ For purposes of the present paper, we assume its validity for such defects. If 


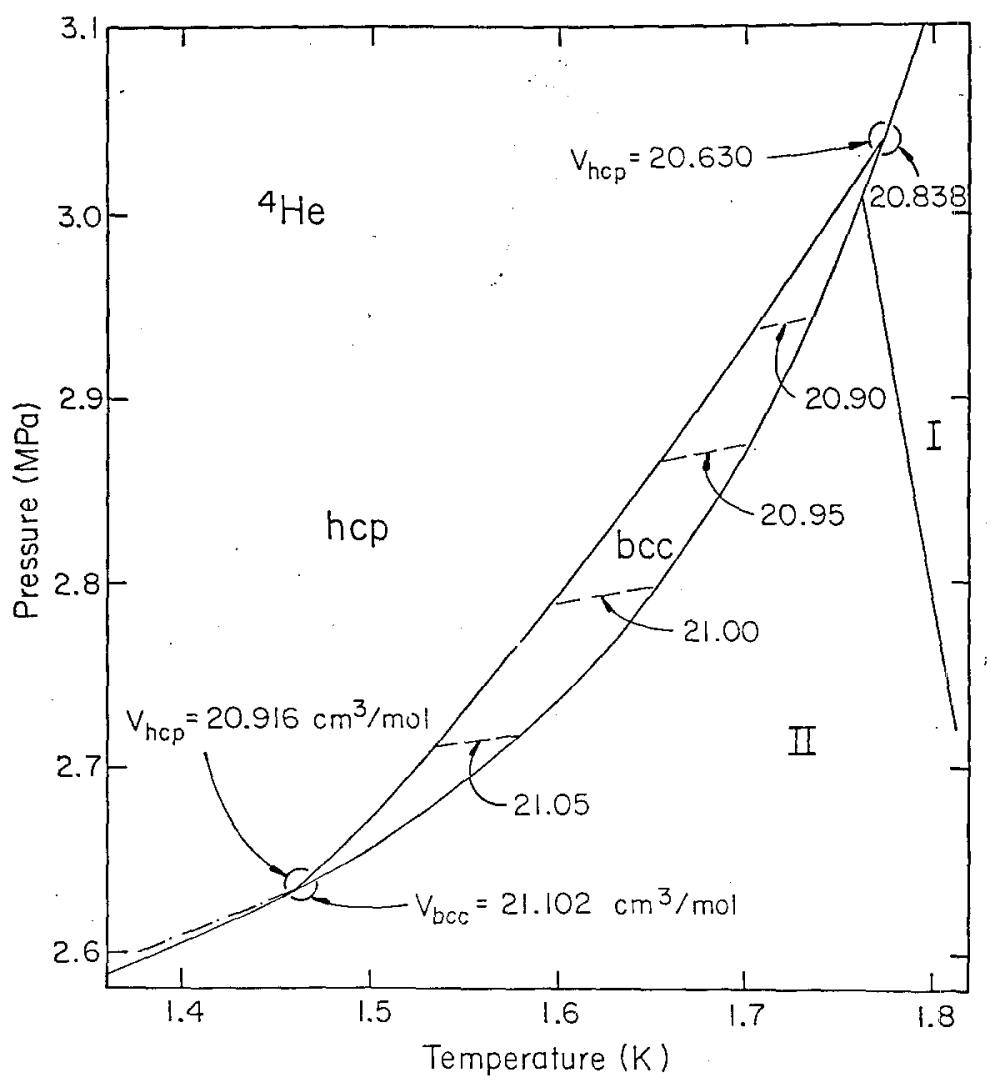

Fig. 1. Pressure-temperature phase diagram of ${ }^{4} \mathrm{He}$ near the bcc phase. ${ }^{12}$ The upper triple point hcp-bcc-I is at $1.772 \mathrm{~K}$ and $3.040 \mathrm{MPa}$. The lower triple point hcp-bcc-II is at $1.463 \mathrm{~K}$ and $2.636 \mathrm{MPa}$. (- -) Isochores in the bcc phase, with the respective macroscopic molar volumes labeled. Molar volumes of hcp and bcc at the upper and lower triple points are also shown. (I, II) The normal and superfluid liquid phases, respectively. (- - ) A metastable bcc-II boundary. ${ }^{13}$

at some time in the future this assumption needs revision, then reinterpretation of our data may be indicated. In any case, the data of the present paper represent a fundamental constraint on any interpretation.

The conventional way to determine $c(\ll 1)$ as a function of temperature consists in measuring simultaneously the macroscopic volume of an unconstrained specimen and its $\mathrm{x}$-ray lattice spacing as the temperature is varied. In such crystals, $c$ is given by

$$
c(T)-c\left(T_{0}\right)=\Delta V / V_{0}-\Delta V_{\mathrm{x}} / V_{\mathrm{x} 0}
$$

Here $\Delta V$ and $\Delta V_{\mathrm{x}}$ are changes of the macroscopic volume of the sample and the x-ray cell volume, respectively, from reference values $V_{0}$ and $V_{\mathrm{x} 0}$, 
measured at a low temperature $T_{0}$. Such a differential method can have greater accuracy than the conditions of Eq. (1), which require absence of specimen porosity, absolute measurements of density, of $x$-ray wavelength, etc.

Because helium solidifies only under external applied pressure, it is not possible to prepare free-standing crystals of helium away from the melting line. However, because solid helium is so compressible, it is easy to hold the macroscopic volume of a sample essentially constant. In this case $\Delta V / V_{0}=0$, and for a cubic crystal Eq. (2) reduces to

$$
c(T)-c\left(T_{0}\right)=-3 \frac{\Delta a}{a_{0}}
$$

Thus, the concentration of vacancies in cubic solid helium may be obtained just by measuring the change in $\mathrm{x}$-ray lattice parameter from a low temperature. In the present paper, we apply both Eqs. (1) and (3) to deduce the thermal vacancy content of bcc ${ }^{4} \mathrm{He}$.

\section{VACANCIES IN SOLID HELIUM}

The energy required to create a vacancy can be related to fundamental energies associated with the solid. Using arguments similar to those of Guyer, ${ }^{16}$ one may describe vacancy formation as a four-step process. First add a particle at fixed temperature and pressure, increasing the volume of the system by $v_{a}$, one atomic volume. The net energy change is

$$
\begin{aligned}
E\left(V+v_{a}, N+1, \eta+1\right)-E(V, N, \eta)+\left(\frac{\delta E}{\delta N}\right)_{V}+\left(\frac{\delta E}{\delta V}\right) v_{a} & =\mu-P v_{a} \\
& =\mathrm{KE}+\mathrm{PE}
\end{aligned}
$$

Here $N$ is the number of particles and $\eta$ the number of lattice sites. The second equality holds because the chemical potential $\mu$ is equal to the sum of kinetic and potential energies plus $P v_{a}$. The second step is to compress the system back to its original volume. This requires an energy approximately equal to $P v_{a}$ :

$$
E(V, N+1, \eta+1)-E\left(V+v_{a}, N+1, \eta+1\right)=-\int_{V+v_{a}}^{V} P d V \approx P v_{a}
$$

Next remove an atom from the interior of the crystal without letting the lattice relax about the vacant site. The energy of the system is lowered by 
2PE + KE: $\mathrm{PE}+\mathrm{KE}$ for the removed particle plus an additional PE for the contribution of the removed particle to the potential wells of all the other particles in the system,

$$
E(V, N, \eta+1)_{\mathrm{ND}}-E(V, N+1, \eta+1)=2 \mathrm{PE}-\mathrm{KE}
$$

The energy

$$
E(V, N, \eta+1)_{\mathrm{ND}}-E(V, N, \eta)=-\mathrm{PE}+P v_{a}
$$

is the energy required to form a vacancy at constant volume if there is no distortion about the vacant site. Finally, let the lattice relax about the vacant site, lowering the energy of the system by $\Delta$,

$$
E(V, N, \eta+1)_{\mathrm{D}}-E(V, N, \eta+1)_{\mathrm{ND}}=-\Delta
$$

Adding up all of these energies, one obtains the constant-volume vacancy formation energy for a localized vacancy, $e_{L}$ :

$$
e_{L}=E(V, N, \eta+1)_{\mathrm{D}}-E(V, N, \eta)=-\mathrm{PE}+P v_{a}-\Delta
$$

A vacancy in the quantum solid heliums differs from a vacancy in a classical solid in that the helium vacancy is much more likely to exchange places with a neighboring atom. Such tunneling changes the vacancy formation energy from a discrete value to a band of energies. In a nearest neighbor tunneling model, with a tunneling matrix element $t$, the result is a band of energies extending from $e_{L}-z t$ to $e_{L}+z t$, where $z$ is the number of nearest neighbor atoms. ${ }^{717}$ The vacancy tunneling frequency $\omega$ is related to the tunneling matrix element by $\omega=t / h$. If the vacancy band is broad enough, it could extend to zero energy, in which case vacancies would be present in the ground state. ${ }^{18}$ Inferences about the vacancy band width in bcc ${ }^{3} \mathrm{He}$ have been made from NMR measurements, ${ }^{19,20}$ but no such evidence can be obtained, unfortunately, for pure ${ }^{4} \mathrm{He}$. Further, firm evidence of groundstate vacancies in either ${ }^{3} \mathrm{He}$ or ${ }^{4} \mathrm{He}$ is lacking, although some recent speculations based upon susceptibility measurements or on NMR in ${ }^{3} \mathrm{He}$ have been made..$^{21,22}$

In the usual treatment ${ }^{23}$ the concentration of vacancies as a function of temperature is given by

$$
c(T)=\exp (-f / k T)=\exp [-(e-T s / k T)]
$$

where $f, e$, and $s$ are, respectively, the free energy, energy, and entropy associated with the formation of a vacancy at constant volume. However, the concentration of vacancies at a given temperature will be different if there is a band of vacancy formation energies rather than a single energy. 
From the Appendix, an expression for the concentration of vacancies, for a vacancy band with density of states $\rho(E)$, is

$$
c(T)=\exp (s / k) \int\{\rho(E) /[\exp (E / k T) \pm 1]\} d E
$$

The plus sign applies if the vacancy is created in a solid of mobile Fermi particles, while the minus sign is for a Bose system. Equation (10) reverts to Eq. (9) in the case where, due to localization, the vacancy band collapses to a single energy, $\rho(E)=\delta(E-e)$, and the vacancy formation energy is much greater than the temperature, $e \gg k T$.

As explained in Section 1, $\mathrm{x}$-ray diffraction provides a means of measuring $c(T)$ directly, independent of theoretical models or of the relaxation occurring around the vacancy. In particular, if measurements are made in solid helium, we expect that Eq. (3) should be valid even if $c(T)$ is given by Eq. (10). It should be remembered, however, that $c\left(T_{0}\right)$ in Eq. (3) will not necessarily be negligible if there are ground-state vacancies. ${ }^{9}$ In any case, if $x$-ray measurements of sufficient accuracy can be made, powerful constraints on $\rho(E)$ can be obtained.

The goal of the present work is to provide more information about the fascinating process of vacancy formation in the solid heliums by determining the energy required to form a vacancy in bcc ${ }^{4} \mathrm{He}$. Comparison of this energy with values for the same quantity in the extensively studied bcc phase of ${ }^{3} \mathrm{He}$ can provide information about the basic energies in the solid heliums and how they depend on isotope.

\section{APPARATUS AND EXPERIMENTAL PROCEDURES}

\subsection{X-Ray Diffractometer/PSD System}

X-ray Bragg scattering measurements on weakly scattering He crystals require specialized diffraction equipment. That used in the present work is described elsewhere. ${ }^{24}$ With it, crystal growth can be observed, crystal quality can be characterized, crystal orientation determined, absolute lattice parameters can be measured, and relative changes in lattice parameter and in Bragg peak intensity can be rapidly followed.

An $\omega$-scan with this system is equivalent to rotating the crystal and leaving the source and detector stationary. Two kinds of data are accumulated during an $\omega$-scan. The "MCA peak" is the peak that accumulates in the multichannel analyzer of the position-sensitive detector (PSD) during the scan. The "SCA peak" is a record of the total number of counts collected in the entire detector as a function of angular position. The SCA peak thus relates source position with scattering intensity and so includes a map of 
the mosaic structure of the crystal, within limitations set by angular divergence of the beam from the source. The MCA peak, on the other hand, averages over the mosaic structure of the crystal, and the MCA peak peak width depends on only the beam divergence, $x$-ray spectral width, and the detector resolution. An MCA peak is shown in Fig. 2.

The method for absolute lattice parameter measurements is described in ref. 24; it involves two scans of the Bragg peak, with opposite directions for the scattering vector. When the two lattice parameters resulting from scanning in both directions are averaged, the accuracy of the measurement is about $300 \mathrm{ppm}$ for Bragg angles in the range $15-20^{\circ}$, which is the case for usable Bragg peaks from solid helium with $\mathrm{Cu} K \alpha$ radiation.

Relative lattice parameter information is derived from shifts in the position of the MCA peak or of the SCA peak. The position of either type of peak is found by subtracting background scattering from the raw data and then computing the centroid of the upper $90 \%$ of the peak that remains. Figure 3 shows an SCA peak and an MCA peak from a data run. A linear fit to the background of each peak has been subtracted so the base of each is at zero counts per channel. In the raw data, the SCA peak has a much

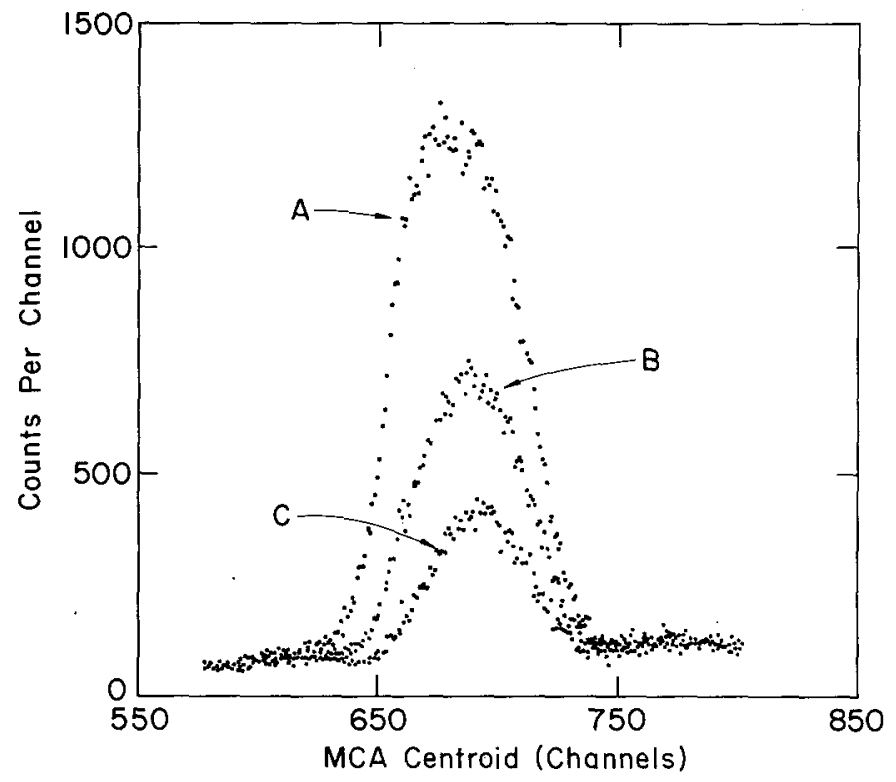

Fig. 2. MCA Bragg peak intensity changes at a phase transition, in this case the hcp to mixed phase. The (110) peaks were obtained with $\mathrm{Cu} K \alpha$ radiation at the following temperatures: (A) 1.600 , (B) 1.621 , (C) 1.630 $\mathrm{K}$. One degree in Bragg angle corresponds to 101.3 channels; the background arising from the cryostat and sample cell is well controlled. 


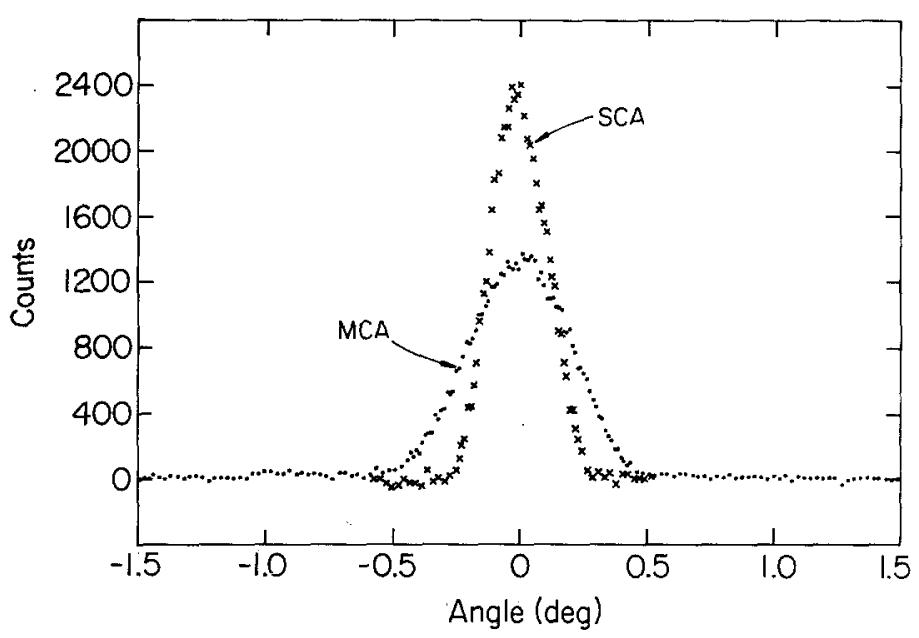

Fig. 3. SCA and MCA Bragg peaks obtained during an $\omega$-scan. The abscissas of the points have been respectively converted from PSD channels (for the $\mathrm{MCA}$ ) and from encoder readings (for the SCA) to degrees, and the centroids of the two peaks are aligned. For clarity, only every other data point of each curve is plotted in the region near zero counts above the subtracted backgrounds.

larger background than the MCA peak, because much of the background is comprised of scattering by the cryostat radiation and vacuum shields. For the data scan pictured, the MCA peak is a little less than twice as wide as the SCA peak. This is typical, but the SCA peak width varies from crystal to crystal, because it depends on the crystal mosaic width, while the MCA peak width is roughly the same for all crystals. Because the SCA peak depends on the crystal position and mosaic, if the crystal rotates by a small amount or the mosaic structure changes slightly between scans, the shape and/or position of the SCA peak will change, but the MCA peak will be unaffected. For our samples of solid helium the most severe changes of the crystal mosaic or position occur between scans when the temperature is changed. For this reason, the MCA data are usually the most reliable indicator of relative lattice parameter changes. If the two sets of data agree, it is likely that the system is working well.

\subsection{Cryogenic Equipment}

The cryostat and ${ }^{3} \mathrm{He}-{ }^{4} \mathrm{He}$ dilution refrigerator used in the present experiments were essentially the same as described previously. ${ }^{25}$ The cryostat is of rigid-tail design, so that the sample cell remains in the same position relative to the diffractometer center independent of the levels of the cryogens. 
Three cylindrical thermal radiation shields, one beryllium and two aluminized Mylar, surround the sample while at the same time allowing $x$-ray access over an angular range of $330^{\circ}$ about the vertical axis and $40^{\circ}$ about the horizontal plane.

The Lucite cell provided a 1.6-mm-diameter, 14-mm-height volume for the helium sample, which was pressurized through capillary connection to an external gas handling system.

\subsection{Procedures}

Direct measurement of vacancy formation in bcc ${ }^{4} \mathrm{He}$ presented us with two experimental difficulties in addition to the challenges of carrying out $\mathrm{x}$-ray diffractometry on solid He. First, the bcc phase does not exist at low temperatures, where the concentration of thermal vacancies should be small. It is therefore impossible to obtain a low-temperature bcc reference lattice parameter from which changes in lattice parameter would give the vacancy concentration directly. The second difficulty is that the fluid phase adjacent to the bcc phase in ${ }^{4} \mathrm{He}$ is superfluid. The thermal conductivity of the superfluid makes it impossible to grow bcc ${ }^{4} \mathrm{He}$ crystals at constant pressure with the experimental configuration of our cryostat for which most of our $\mathrm{x}$-ray crystal growing experience is available; superfluid in the specimen fill line conducts too much heat from warm parts of the cryostat to allow the sample to be cooled below the lambda-line temperature at whatever pressure one is attempting to grow a crystal.

To circumvent this problem, crystals were grown at constant volume. The cell was initially filled at a temperature and pressure above the upper triple point (Fig. 1), then the fill line was plugged. The temperature at the top of the cell was slowly reduced, and eventually hcp solid began growing in the cell. Typical behavior for the thermometer at the bottom of the cell is shown in Fig. 4, during production of a bcc crystal. Measurements of absolute lattice parameters on such bcc crystals were then made along the melting line. (A sharp blip on the thermometer appeared if the sample moved off the melting line into a totally solid condition.) For extensive melting line data from a given sample, it was desirable to fill the cell at a molar volume of $21 \mathrm{~cm}^{3} /$ mole or greater.

On the other hand, application of Eq. (3) requires a low-temperature reference state, which required filling of the cell at a molar volume at about $20.9 \mathrm{~cm}^{3} /$ mole (see Fig. 1). We succeeded in one case.

\section{DATA}

Of $61{ }^{4} \mathrm{He}$ specimens prepared in the present work, 22 had accessible Bragg reflections of sufficient quality that data-taking was deemed 


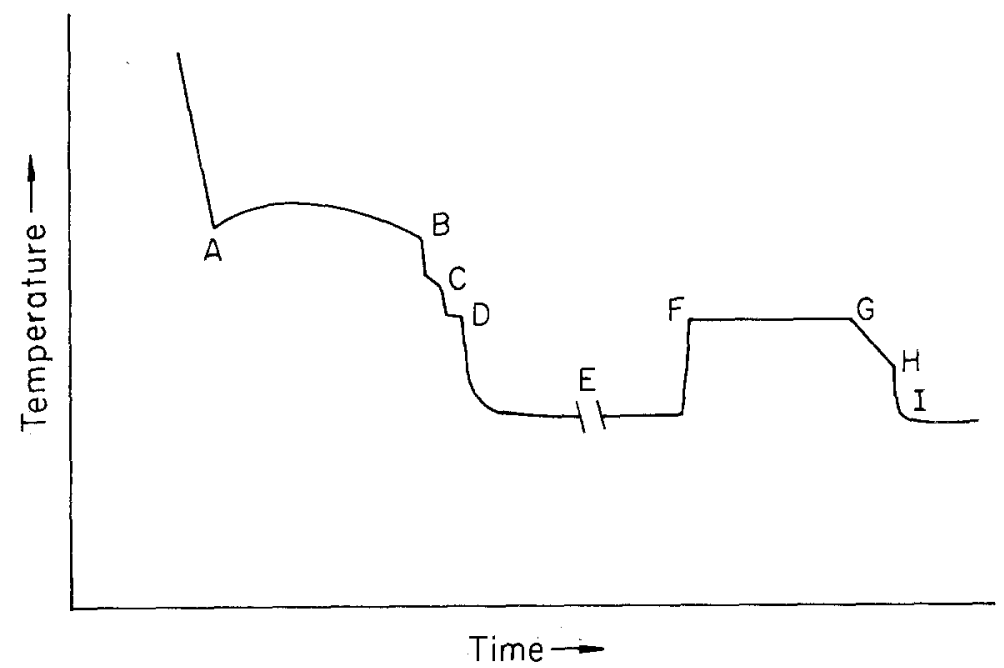

Fig. 4. Schematic crystal growth curve at constant molar volume, as heater power at cell bottom was reduced. (A-B) The hep crystal growth, (C) kink at about the hcp-bcc-I triple point, (D) kink at about the bcc-I-II triple point, (E) constanttemperature region, lasting from 2 to $10 \mathrm{hr}$, in which solid is hcp, as verified by Laue photographs, (F) warming to about bcc-I-II point, (G-I) cooling to temperature near $\mathrm{E}$, with Laue photographs after point I showing solid to be bcc.

worthwhile. Of these, eight provided no useful information-some were unstable and some were subjects of unsuccessful attempts to obtain data off melting in the bcc and hcp phases. Data from the remaining 14 specimens, with some comments, are summarized below.

\subsection{Melting Line Data}

These data consist of measurements of absolute lattice parameters $a$ of 13 bcc crystals in equilibrium with the superfluid. The crystals were grown using the method described in Section 2 with starting pressures usually within a few tens of $\mathrm{kPa}$, but in a few cases as much as $400 \mathrm{kPa}$, above the upper triple point pressure of the bcc phase. Details of crystal growth are presented in Section 2.3.

The majority of the samples studied remained on the melting line (i.e., at least some fluid remained in the cell) at all temperatures. In such a sample the bcc spots that were found after the solid transformed from the hcp phase during crystal growth remained present as the sample was cooled. Samples that remained on the melting line at all temperatures could be used to measure bcc lattice parameters at melting throughout the temperature range of the bcc phase. 
Melting line data are shown in Fig. 5 for 13 specimens. The open symbols show $a$ measured by scanning the x-ray source in one direction, while the solid symbols show resulting averages of $a$ values at a given temperature when there was time to scan the source both clockwise (CW) and counterclockwise $(\mathrm{CCW})$. Fifteen of the 40 data points are from one particularly high-quality specimen (triangles). None of the other 12 specimens is represented by more than four points. The smooth curves on Fig. 5 are derived from bulk density data, as explained below in Section 5.1.

The biggest factor limiting the accuracy of these absolute lattice parameter data was rotation of the crystals. That is, the crystal (in contact with superfluid) would sometimes rotate through a small angle about the cylindrical axis of the cell between the times that the two Bragg peaks necessary for an absolute measurement were recorded. A rotation of only $0.01 \mathrm{deg}$ corresponds to a shift in $a$ of $325 \mathrm{ppm}$. For a stable specimen the verified accuracy of our method is $300 \mathrm{ppm} .{ }^{24}$ On a few occasions the apparent shifts in $a$ were so large that it was obvious that rotation had taken place; such a data point was discarded. In other cases shifts were not obvious, although the data scatter was above the expected 300-ppm level. We chose to discard a data point if the $\mathrm{CW}$ and $\mathrm{CCW}$ scans disagreed by $1800 \mathrm{ppm}$ or more, or if a preceding or following scan in one direction only at the same temperature disagreed by more than $1800 \mathrm{ppm}$; these criteria eliminated 8 of 69 measurements. A look at Fig. 5 reveals that small rotations must have occurred during the measurement of many of the remaining

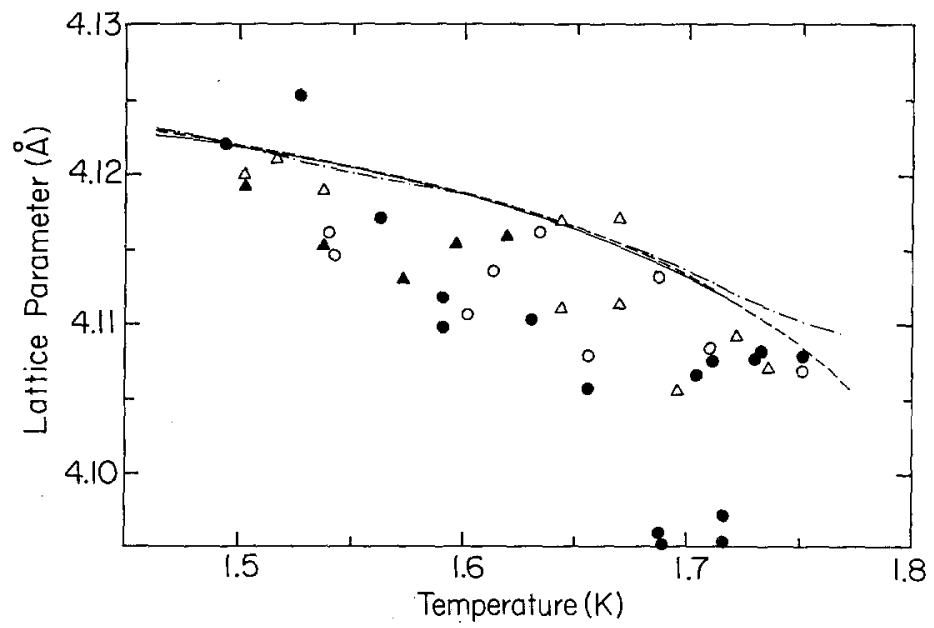

Fig. 5. Absolute lattice parameter measurements on bcc ${ }^{4} \mathrm{He}$ in contact with superfluid. Symbols are explained in Section 4.1. The smooth curves are derived from bulk measurements refs. 12,13 , and 27 . 
lattice parameters. In the absence of any unbiased method for identifying the contributions of such rotations or of controlling them for the bcc crystals in contact with superfluid, however, we suppose that such rotations, in both directions, introduced a random distribution of errors that will largely cancel one another when smoothed.

A sample could usually be cooled several mdeg below the lower triple point of the bcc phase $\left(T_{L}\right)$ without the solid changing from bcc to hcp. This was verified by the continued presence of bcc Bragg peaks in the detector and by the absence of hcp spots on Laue pictures. Eventually the solid would transform to hcp. This was marked by a sharp rise in the temperature of the thermometer (G1) at the bottom on the cell to $T_{L}$, followed by a slower drop back to the original temperature. After this transition, Laue pictures confirmed that the sample was hcp. There was similar metastable behavior of the hcp solid, but usually over a smaller temperature range, when the sample was warmed through $T_{L}$. Again, this hcp to bcc transition was marked by a blip in thermometer G1 and directly confirmed by Laue pictures. These metastabilities observed directly, through $\mathrm{x}$-ray identification of the solid phases, confirm the earlier observations of Grilly, ${ }^{12}$ Hoffer and co-workers, ${ }^{13}$ and Efimov and Mezhov-Deglin. ${ }^{26}$ One can supercool the bcc phase and superheat the low-density hcp phase; it appears easier to nucleate bcc phase from hcp than the reverse.

\subsection{Data off Melting}

Originally, an hcp crystal was grown at conditions somewhat above the hcp-bcc-I triple point, but at a molar volume in the plugged cell such that a bcc crystal subsequently filled the cell at $1.735 \mathrm{~K}$. Data then taken in the bcc phase are shown in Fig. 7; the bcc phase extended from about 1.735 to $1.70 \mathrm{~K}$. At temperatures below $1.70 \mathrm{~K}$, the sample (at constant macroscopic volume) went through the transition to a mixed hcp-bcc phase, and it returned as the same function of temperature; this hcp Bragg peak was already shown in Fig. 2. The circles in Fig. 7 are from several different warming and cooling runs through the bcc-mixed transition; below $1.70 \mathrm{~K}$ the bcc lattice parameter is depressed as the fraction of the cell filled by mixed phase increases. Judged by variations in the intensity of the hcp Bragg peak, the mixed phase for this specimen extended down to $1.595 \mathrm{~K}$.

When the sample was warmed above $1.735 \mathrm{~K}$, however, the bcc parameter $a$ changed irreversibly. When cooled back below $1.735 \mathrm{~K}, a$ again was a reproducible function of temperature (shown as crosses). Apparently $1.735 \mathrm{~K}$ was the melting temperature for this sample, above which the plug in the fill line slipped, allowing the higher pressure in the external fill line to compress the specimen compared to the case of the original sample. 


\section{TABLE I}

X-Ray Lattice Parameter Measurements on the Sample Described in Section 4.2, with a Nominal Melting Temperature of $1.735 \mathrm{~K}$, Confined at Constant Macroscopic Volume ${ }^{a}$

$\left.\begin{array}{cccc}\hline \text { Structure } & (h k l) & T, \mathrm{~K} & a, \AA \\ \hline \text { HCP } & 10 \overline{1} 0 & 0.822 & 3.6621 \pm 0.0009 \\ & & 0.921 & 3.6621 \pm 0.0009 \\ \text { BCC } & 110 & 1.716 \\ & & 1.727 & 4.1025 \pm 0.002 \\ & & 1.731 & \\ & & 1.751\end{array}\right\}$

${ }^{a}$ From the hcp data the low-temperature reference molar volume is $V_{0}=20.900 \mathrm{~cm}^{3}$.

Even in the narrow 35-mdeg range of pure bcc phase in this specimen one can apparently see thermal vacancy generation at the two respective slightly different molar volumes of the two pressures. Considering the estimated uncertainties of the individual data points, however, one needs a wider range of temperature before a reliable estimate of the thermal vacancy formation energy is produced. This we do next.

To apply Eq. (3) one needs a low-temperature reference volume, and this is available in such a specimen confined at constant volume, even though the low-temperature crystal structure is hcp (Fig. 1). After the relative lattice parameter measurements on bcc (110) were taken (Fig. 7), the sample was cooled for two absolute lattice parameter measurements in the hcp phase; the crystal was stable and these had the expected 300-ppm accuracy. Finally, the sample was warmed for absolute measurements of $a$ in the bcc phase. Unfortunately, temperature control in the cryostat was deteriorating, so that these measurements of $a$ have an estimated accuracy of $500 \mathrm{ppm}$. The results are shown in Table I.

\section{ANALYSIS AND DISCUSSION}

We discuss our data both on and off the bec melting curve, and show they give the same value for the free energy of vacancy formation in bcc ${ }^{4} \mathrm{He}$. Some comparisons to inferences made from other, indirect, techniques are then made. Finally, bcc ${ }^{4} \mathrm{He}$ and ${ }^{3} \mathrm{He}$ are compared, and an unresolved puzzle is revealed.

\subsection{Melting Line Data}

Bulk molar volumes of bcc ${ }^{4} \mathrm{He}$ at its phase boundary with the superfluid have been measured in several studies. ${ }^{12,13,27}$ The curves in Fig. 6 are plots of $\left(2 \mathrm{~V} / N_{\mathrm{A}}\right)^{1 / 3}$, where $N_{\mathrm{A}}$ is Avogadro's number, computed from the values 


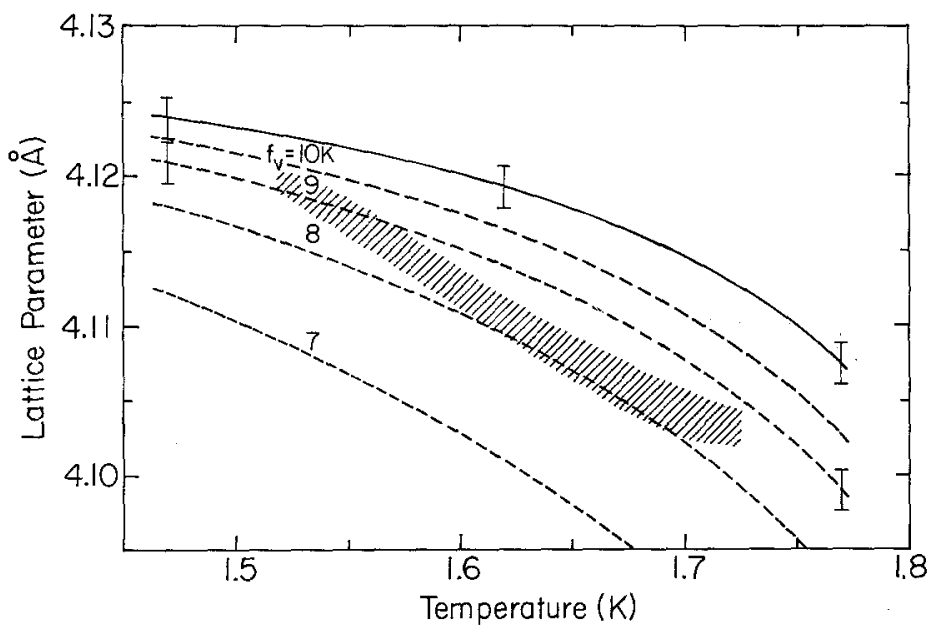

Fig. 6. Comparison of bulk and $\mathrm{x}$-ray volumes in $b \mathrm{cc}^{4} \mathrm{He}$. The cross-hatched region is the envelope of low-order polynomial fits to the present $x$-ray data for the central $80 \%$ of the temperature range of the fits. (-) From bulk data of ref. 13 with $0.1 \%$ added to the volumes. The error bars represent an uncertainty of $\pm 0.1 \%$ in the measured bulk volumes. (- -) Calculations of the $\mathrm{x}$-ray lattice parameter from the bulk volumes represented by the solid line and the vacancy formation free energies $f$ indicated.

of the bulk volume $V$, reported in these studies. Clearly, the great majority of the X-ray points fall below the bulk volume lines. This is consistent with the existence of thermal vacancies at melting.

The accuracy of the vacancy concentration determined using Eq. (1) depends upon the absolute accuracy of both the $\mathrm{x}$-ray and bulk volume data. The two most recent determinations ${ }^{12,13}$ of the bulk volume at melting agree with each other quite well. In two articles by the Berkeley group ${ }^{13,28}$ the precision of their bulk molar volumes is claimed to be $200 \mathrm{ppm}$; indeed, the values are close to those of Grilly. ${ }^{12}$ Neither Grilly nor the Berkeley group measured absolute values themselves; rather, each used a (different) single $P V T$ point from the absolute data of Grilly and $\mathrm{Mills}^{27}$ as a reference. The Berkeley group indicates ${ }^{28}$ that their assigned volumes would be increased by an average of $0.2 \%$, with no systematic dependence on volume, if they used instead as a reference the liquid ${ }^{4} \mathrm{He}$ data of Hill and Lounasmaa. ${ }^{29}$ For purposes of the present analysis, we use the volumes of the Berkeley group ${ }^{13}$ with $0.1 \%$ added to them, and we assign an uncertainty of $0.1 \%$ to the bulk volumes so used.

To smooth the $\mathrm{x}$-ray data shown in Fig. 5, low-order (first through fourth) polynomials were fitted to the lattice parameter data with the (solid) points obtained by scanning both $\mathrm{CW}$ and $\mathrm{CCW}$ given double weight. The 
fits were performed with no constraints on the functions at the temperature extremes. We show in Fig. 6 the results as a hatched region, which is the envelope of the four polynomial fits in the central $80 \%$ of the temperature range. In Fig. 6 the solid line is the bulk volumes ${ }^{13}$ as described above.

The thermal vacancy concentration is well approximated by Eq. (9) if the vacancy band is narrow. Using Eqs. (1) and (9), one can calculate what the lattice parameter at melting would be for a given choice of bulk volume $V$ and vacancy free energy $f$. The dashed lines of Fig. 6 represent a series of such calculated values for the series of $f$ values indicated. Comparing these curves to the hatched $\mathrm{x}$-ray region, one sees that a reasonable value for $f$ is $9 \pm 1 \mathrm{~K}$. Over such a small molar volume range, we take $f$ to be constant to within the uncertainty of the present measurements.

\subsection{Data off Melting}

Equation (3) can be applied to the present data of Table I, with the reasonable assumptions that hep ${ }^{4} \mathrm{He}$ is ideally close-packed ${ }^{1}$ and there are negligible ground-state vacancies in the hep phase. For an average of the bcc values, one finds a thermal vacancy concentration of about $7 \times 10^{-3}$ at the average high temperature, and from Eq. (9) one finds a corresponding value of $f=8.5 \mathrm{~K}$. Taking the estimated possible uncertainties in the lattice parameters of the two phases as the limits of possible error, one finds a total range for the deduced value of $f$ to lie between 7.9 and $9.6 \mathrm{~K}$.

Because only $\mathrm{x}$-ray measurements were used to obtain these values and all measurements were made on the same solid sample, these values are independent of those in the previous section. The fact that the two methods are in agreement is useful confirmation of each. Note that each method is direct, i.e., counts vacancy sites independent of secondary characteristics of the vacancy.

The above analysis depends upon an average of the measured lattice parameters in the bcc phase. Examination of Fig. 7 does show an apparent temperature dependence in the narrow bcc domain, one actually greater than that to be expected for a free energy value near $9 \mathrm{~K}$. This situation, in a very narrow region near the melting point, is reminiscent of that in some data of Heald and co-workers for bcc ${ }^{3} \mathrm{He} .{ }^{5}$ In both cases, the accuracy of the x-ray measurements is somewhat limited. We therefore regard it as best to use the largest possible temperature interval, and as unjustified to take any relation more intricate than Eq. (9), for the analysis of the current data.

\subsection{Vacancy Properties Inferred from Other Techniques}

The literature reports four types of experiments that have been interpreted to yield information about vacancy formation, or at least vacancy 


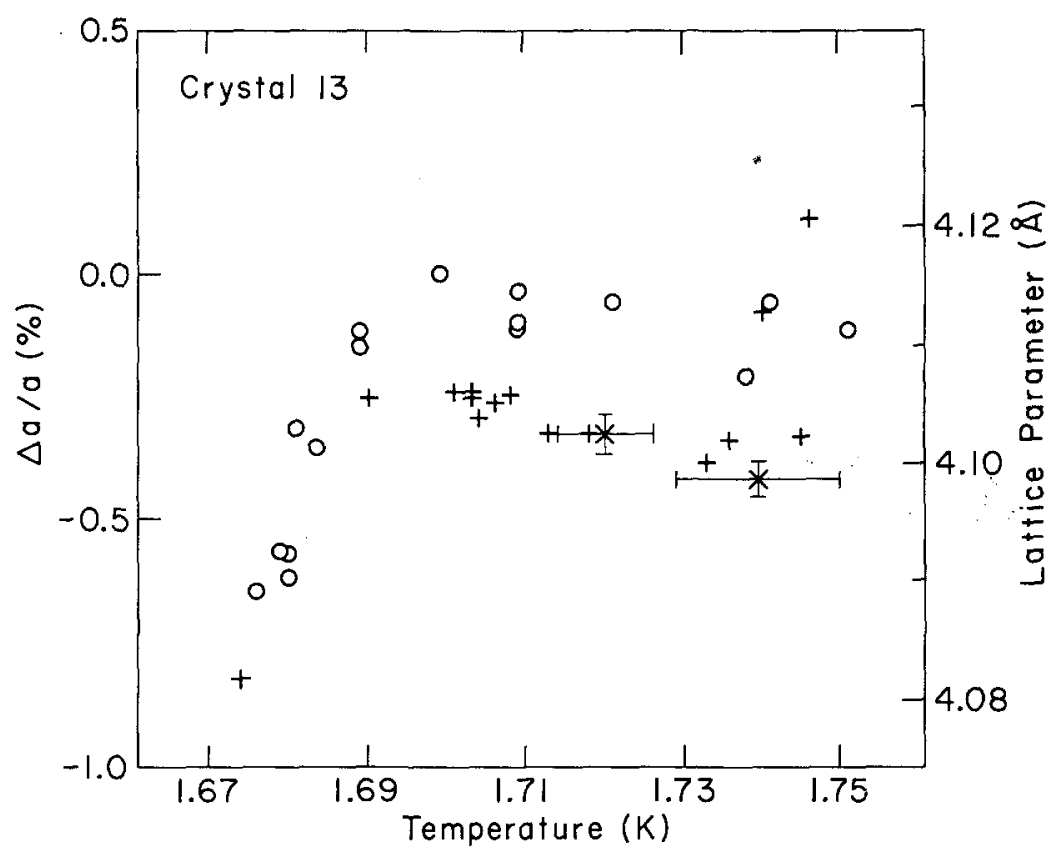

Fig. 7. BCC phase measurements for a specimen off melting $(+)$ Measurements made after the specimen was warmed above $1.735 \mathrm{~K}$. $(x)$ Two absolute measurements made after study of the hcp phase. Vertical error bars shown are $300 \mathrm{ppm}$ in lattice parameter, which is somewhat smaller than the actual case for these bcc phase measurements; horizontal error bars show the temperature change during each of the absolute measurements, during which the specimen was warming.

diffusion, in the solid heliums. The four types of study are (1) NMR, (2) ion mobility, (3) ultrasonic, and (4) calorimetric. Few of these have very directly addressed the question of bcc ${ }^{4} \mathrm{He}$, which exists in such a small PVT domain. But it is worthwhile summarizing available results, both for the intrinsic interest and for the introduction it provides to a puzzle about the structure of the vacancy in bcc solid heliums.

Because ${ }^{4} \mathrm{He}$ has no nuclear spin, NMR studies involve monitoring the motion of ${ }^{3} \mathrm{He}$ impurities in the temperature regime where such motion is thought to be dominated by movement into vacant atomic sites. The introduction of ${ }^{3} \mathrm{He}$ also expands the domain of bcc solid. Results have been reported for ${ }^{3} \mathrm{He}$ concentrations of $2.17 \%,{ }^{30} 1.95 \%,{ }^{31}$ and $0.75 \%{ }^{30}$ and the smaller amounts $2.5 \times 10^{-3}$ to $1 \times 10^{-4}$ in the bcc phase. ${ }^{32}$ While there is no precise agreement among the results of the different NMR studies, the present $\mathrm{x}$-ray formation free energy $(9 \pm 1 \mathrm{~K})$ is about equal to the average value of the NMR activation energies. If the motion of ${ }^{3} \mathrm{He}$ atoms 
in ${ }^{4} \mathrm{He}$ is mediated by vacancy motion, the diffusion activation energy is equal to the vacancy formation energy plus the vacancy migration energy. The migration energy is zero if vacancies move by tunneling, but is equal to the barrier height if vacancies diffuse classically by jumping over barriers. The formation free energy and entropy are related as in Eq. (9); if $T s \ll e$, then the fact that the NMR and $x$-ray energies are not measurably different is consistent with the notion that vacancies move by tunneling in $b c c{ }^{4} \mathrm{He}$. This would be similar to the situation in bcc ${ }^{3} \mathrm{He}^{5,6}$

Ion mobility has been reviewed by $\mathrm{Dahm},{ }^{33}$ who describes the various ways that vacancies have been ascribed a role in the phenomena. A particular study of bcc ${ }^{4} \mathrm{He}$ has been made by Golov and co-workers, ${ }^{34}$ who find that the activation energies for ion mobilities in this phase are, respectively, $10 \mathrm{~K}$ for negative ions and $15 \mathrm{~K}$ for positive ions. In this respect, the situation is similar to that of $b_{c c}{ }^{3} \mathrm{He}$ for molar volumes greater than $21.5 \mathrm{~cm}^{3}$. The negative ion at these larger molar volumes is thought to be surrounded by a small cavity; from the correspondence between the activation energies, motion of the cavity in these conditions is thought to be vacancy-assisted. ${ }^{33}$ This would then also seem to be the case in bcc ${ }^{4} \mathrm{He}$.

Detailed calorimetric study of bcc ${ }^{4} \mathrm{He}$ by Hoffer and co-workers ${ }^{13}$ gave values for many thermodynamic properties, including constant-volume heat capacity $C_{V}$. The "most prominent feature" of their $C_{V}$ data was an (unexplained) "pretransition anomaly-a gradual increase in $C_{V}$ in the $20 \mathrm{mK}$ interval just below the melting temperature." Neglecting this puzzle, one notes that these authors found many parallels in other high-temperature thermodynamic properties with bcc ${ }^{3} \mathrm{He}$. With the results of the current $\mathrm{x}$-ray study in hand, one notes an additional parallel, namely, a very large expected contribution to $C_{V}$ if the vacancies were treated as localized, as in the studies on bcc ${ }^{3} \mathrm{He}$ of Heald and co-workers. ${ }^{6}$ In view of the pretransition anomaly and the limited accuracy of this x-ray vacancy work, in particular its inability to resolve precisely the possible temperature and volume dependence of vacancy properties in the narrow domain of bcc ${ }^{4} \mathrm{He}$, caution in interpretation seems appropriate. For the present, it can be said that the results are consistent, in the cases of both bcc ${ }^{4} \mathrm{He}$ and large molar volume bcc ${ }^{3} \mathrm{He}$, with a nonlocalized model for the vacancy. The arguments for ${ }^{4} \mathrm{He}$ are similar to those already given for ${ }^{3} \mathrm{He} .{ }^{6}$

\subsection{Vacancy Relaxation Energy in $\mathrm{BCC}{ }^{4} \mathrm{He}$ and ${ }^{3} \mathrm{He}$}

Because the bcc phase of ${ }^{3} \mathrm{He}$ has been so much studied, it is of interest to see how the formation free energy in bcc ${ }^{4} \mathrm{He}$ compares to that in bcc ${ }^{3} \mathrm{He}$. The value of $f$ in ${ }^{3} \mathrm{He}$ at the same molar volume and in the same crystal structure studied here is, by interpolation in the data of Heald, ${ }^{6} 10.8 \mathrm{~K}$. 
This is about $20 \%$ larger than the present ${ }^{4} \mathrm{He}$ result. Grigor'ev and coworkers ${ }^{30}$ note a similar difference in the same direction between the NMR activation energies measured by them in bcc mixtures of ${ }^{3} \mathrm{He}$ in ${ }^{4} \mathrm{He}$ and the NMR energies measured by Reich $^{35}$ in pure ${ }^{3} \mathrm{He}$ at nearly the same molar volume. They point out that identical molar volumes in pure ${ }^{3} \mathrm{He}$ and in the solutions correspond to different pressures. They therefore attribute the decrease in the activation energy in the mixtures to the decrease in the contribution to $f$ made by the terms that account for the energy needed to expand the crystal when vacancies are formed.

This idea can be quantified, and tentative inferences concerning relaxation about vacancies in the bcc phases of ${ }^{3} \mathrm{He}$ and ${ }^{4} \mathrm{He}$ made, by the arguments of Eqs. (4)-(8) in Section 2. Equation (8) is an expression for the constant-volume vacancy formation energy $e$. The present measurements, and those of Heald and co-workers, ${ }^{6}$ are of the free energy, $f=e-T s$. Various arguments ${ }^{6,36}$ have been put forward which conclude that it is reasonable to suspect that $T s$ is small compared to $e$, and therefore $f$ and $e$ are not very different from one another; in what follows we assume this. Values for the various energies in the respective isotope crystals are listed in Table II. ${ }^{37,39}$ The difference in $P v_{a}$ between the two isotopes is in the same direction as the difference in formation energies, but the difference in the $P v_{a}$ values is vastly greater.

The large $P v_{a}$ terms for both isotopes, and in particular for ${ }^{3} \mathrm{He}$, must be compensated by very large values of $\Delta$, the constant-volume distortion energy, if one uses Eq. (8). The $\Delta$ value for ${ }^{3} \mathrm{He}$ is about $65 \%$ larger than for ${ }^{4} \mathrm{He}$. One should recall, in examining this rather surprising result, that very simple arguments were used to obtain Eq. (8) and that effects of vacancy delocalization were ignored. It is possible that the procedure outlined, which

TABLE II

Terms Contributing to the Vacancy Formation Energy in $\mathrm{BCC}^{3} \mathrm{He}$ and ${ }^{4} \mathrm{He}$ at about $21 \mathrm{~cm}^{3} / \mathrm{mole}^{a}$

\begin{tabular}{rrrrc}
\hline & $e, \mathrm{~K}$ & $P v_{a}, \mathrm{~K}$ & $-\mathrm{PE}, \mathrm{K}$ & $\Delta=-e-\mathrm{PE}+P v_{a}, \mathrm{~K}$ \\
\hline${ }^{4} \mathrm{He}$ & 9.0 & 7.1 & 20.4 & 18.5 \\
${ }^{3} \mathrm{He}$ & 10.8 & 20.1 & 21.3 & 30.6 \\
\hline
\end{tabular}

${ }^{a} e$ is the constant-volume formation energy of the left-hand side of Eq. (8). All other terms are identified in the text leading up to Eq. (8). The values used for $e$ are the $f$ values measured in the present work for ${ }^{4} \mathrm{He}$ and in ref. 6 for ${ }^{3} \mathrm{He}$. The potential energy for ${ }^{3} \mathrm{He}$ is from the calculation of ref. 37. The potential energy for ${ }^{4} \mathrm{He}$ was obtained by subtracting an estimate of the kinetic energy (equal to three-fourths of the Debye energy of ref. 38) from a value of the ground-state energy extrapolated from the values measured in hcp ${ }^{4} \mathrm{He}$ (ref. 39). The respective estimated errors in the $e$ and the PE values are about $1 \mathrm{~K}$ each, so from this analysis the relaxation energies for vacancies in the two isotopes differ significantly. 
considers the energies of individual atoms, is not meaningful in a solid, where collective effects are so important. It is also possible that some of the other energies entered in Table II are incorrect. For example, it is to be expected in a static crystal that at the same molar volume the potential energy per atom will be independent of isotopic mass; the inferred values of the potential energies listed in the table, obtained by the methods stated in the footnote, are indeed essentially equal. But these quantum solids have considerable momentum distributions of the atoms, ${ }^{2}$ corresponding to considerable nonlocalization of the atoms about their sites. Each atom sits in an effective potential minimum determined in a self-consistent way through the distribution of positions of other atoms in the crystal. These effective potentials need not be strictly the same in the two isotopes.

Given this large apparent difference in the relaxation energies for vacancies in the two isotopes, it is of interest to look for any other comparisons. One simplicity can be found in the apparent free energy of thermal vacancy formation at the melting temperature. As shown in Fig. 8, the

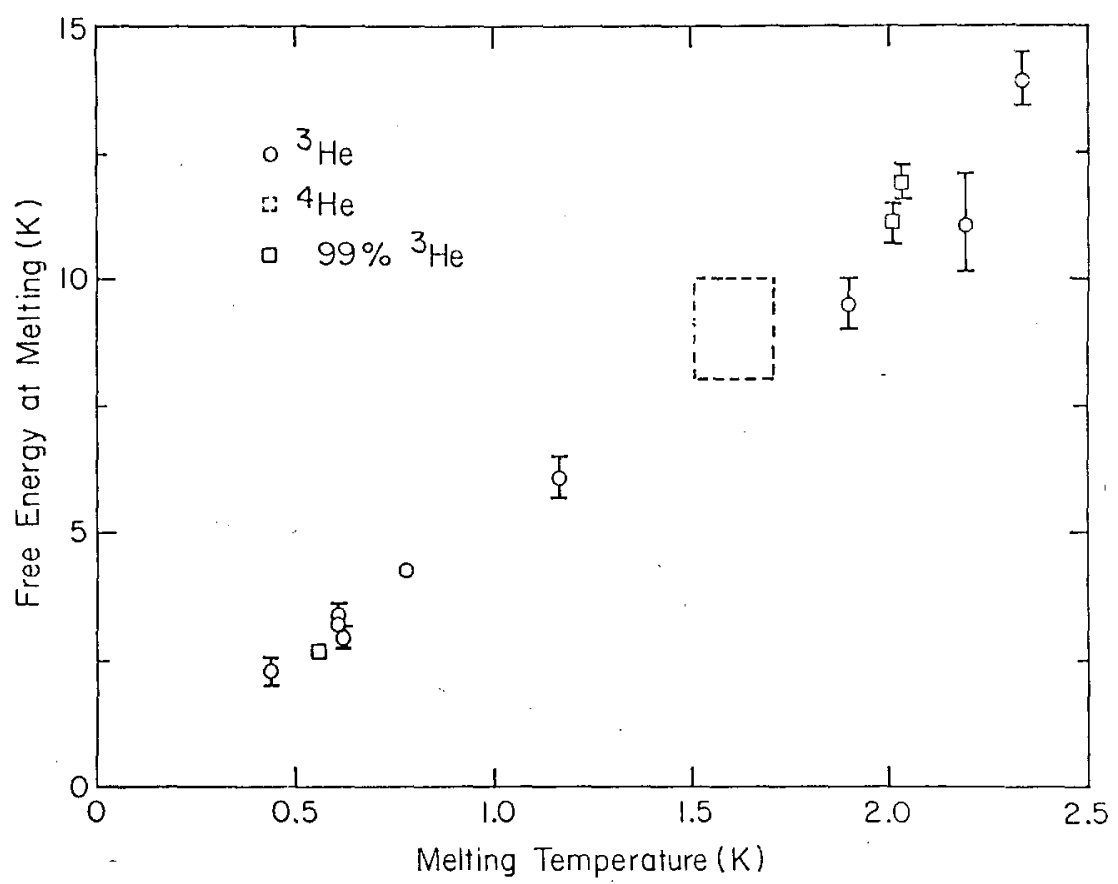

Fig. 8. For various bcc solid heliums of different (nearly pure) isotopic compositions, the apparent free energy of thermal vacancy formation at the melting temperature is proportional to the melting temperature, that is, the vacancy concentration is about a constant number along the bcc melting line. Present work is ${ }^{4} \mathrm{He}$ data; the ${ }^{3} \mathrm{He}$ data are from refs. 5 and 6. 
inferred free energies for both $b c c{ }^{3} \mathrm{He}$ and $b c c{ }^{4} \mathrm{He}$ fall on the same linear relation when plotted versus the melting temperature.

\subsection{Vacancy Free Volume of Formation}

Another way to compare the distortions caused by vacancies in ${ }^{3} \mathrm{He}$ and ${ }^{4} \mathrm{He}$ is to compare the respective free volumes of formation $v$. Heald and co-workers ${ }^{6}$ have calculated $v$ in bcc ${ }^{3} \mathrm{He}$ as a function of molar volume by a number of different means. The free volumes determined from vacancy contributions to the compressibility and to the coefficient of thermal expansion agree well with each other and with those found using the model of Holder and Granato. ${ }^{40}$ Unfortunately, the bcc phase of ${ }^{4} \mathrm{He}$ does not exist in a wide enough temperature range to allow accurate determination of the vacancy contributions to the compressibility or to the thermal expansivity. However, it should be feasible to obtain an estimate of $v$ using the model of Holder and Granato. They give the expression

$$
v=g\left(G^{\prime} / G-1 / B\right)
$$

where $G$ is the shear elastic constant, $G^{\prime}$ its pressure derivative, $B$ the bulk modulus, and $g$ the (constant-pressure) vacancy formation free energy. For given conditions of $P, V$, and $T$, the Gibbs free energy $g$ is equal to the free energy $f$ determined in the present work. $G$ and $B$ can be obtained from the elastic constants determined by Greywall ${ }^{38}$ from sound velocity measurements. Greywall reports elastic constants at only one molar volume and does not specify the pressure dependence, although some sound velocities were measured as a function of pressure. If the pressure dependence of $B$ and $G$ could be inferred, then $v$ could be estimated for bcc ${ }^{4} \mathrm{He}$. Comparison of the respective $v$ values for bcc ${ }^{3} \mathrm{He}$ and ${ }^{4} \mathrm{He}$ could be of interest to test the relationship between vacancy energies and vacancy volumes predicted by Eshelby's continuum theory. ${ }^{14}$

\subsection{Relation to $\mathrm{HCP}{ }^{4} \mathrm{He}$}

In ${ }^{3} \mathrm{He}$ it appears that a transition to a different crystal structure does not much change the value of the vacancy formation energy if the values are compared at the same molar volume. That is, in ${ }^{3} \mathrm{He}$ the single value directly measured in the hcp phase ${ }^{6}$ appears to fall on a smooth extrapolation of the values measured over a range of larger molar volumes in the bcc phase. It is of interest to examine the situation for hcp ${ }^{4} \mathrm{He}$ near $21 \mathrm{~cm}^{3} /$ mole.

A direct measurement by $\mathrm{x}$-ray diffraction has been made by Fraass and co-workers, ${ }^{41}$ who give a formation energy in the range $8.2-8.6 \mathrm{~K}$ for an apparent entropy in the range -0.2 to 0 . This agrees with the present bcc value within the estimated errors, and therefore indicates a similarity 
between ${ }^{3} \mathrm{He}$ and ${ }^{4} \mathrm{He}$ in this respect. If, to turn the argument around, one takes this similarity for granted, the agreement is an indirect confirmation of the present measured values for the formation energy in bcc ${ }^{4} \mathrm{He}$.

For many years vacancies have been freely invoked as contributors to ion and impurity mobilities in the heliums, so the present direct $x$-ray results can be used to point out some cautions. We remind the reader that in bcc ${ }^{4} \mathrm{He}$ and in large molar volume $b c c{ }^{3} \mathrm{He}$ it is the negative-ion activation energies that correspond to the $\mathrm{x}$-ray values, not the positive-ion values as in the hcp ${ }^{4} \mathrm{He}$ phase near $21 \mathrm{~cm}^{3} .{ }^{34}$

\section{APPENDIX}

Suppose that the atoms are mobile, the vacancy energies $E$, form a band with density of states $\rho(E)$, and there are no restrictions on where the density of states may be finite. When $E<k T$, it is necessary to take statistics into account. We assume that vacancy concentrations are small, so that vacancies in ${ }^{3} \mathrm{He}$ can be treated as a gas of noninteracting fermions and vacancies in ${ }^{4} \mathrm{He}$ as a noninteracting gas of bosons. One can define a grand partition function

$$
Z_{V}=\left\{\sum_{N} \sum_{j} \exp -\left[\beta\left(E_{j}-\mu N\right)\right]\right\}^{w}
$$

where $N$ is the number of vacancies, $\beta=1 / k T, j$ denotes the set of all states with fixed number of vacancies $N, \mu$ is the chemical potential, and $w$ is the number of states of the rest of the crystal associated with each vacancy state. The thermodynamic potential is

$$
\Omega=-k T \ln Z_{V}
$$

and the total number of vacancies is

$$
N=(\partial \Omega / \partial \mu)_{T, V}
$$

If the state $i$ has energy $e_{i}$ and $n_{i}$ vacancies in it, Eq. (A1) becomes

$$
Z_{V}^{1 / w}=\sum_{\left\{n_{i}\right\}} \exp \left[-\beta\left(\sum e_{i} n_{i}-\mu \sum n_{i}\right)\right]=\prod_{i} \sum_{n_{i}} \exp \left[-\beta\left(e_{i} n_{i}-\mu n_{i}\right)\right]
$$

Now in ${ }^{3} \mathrm{He}$ the occupation number $n_{i}$ has two possible values, 0 or 1 , and Eq. (A4) reduces to

$$
Z_{V}^{1 / w}=\prod_{i}\left\{1+\exp \left[-\beta\left(e_{i}-\mu\right)\right]\right.
$$

From Eqs. (A2) then (A3) one obtains

$$
N=w \sum_{i}\left[e^{\beta\left(e_{i}-\mu\right)}+1\right]^{\sim 1}
$$


This can be simplified: (a) $w$ is related to the nonconfigurational entropy $s$ associated with formation of a vacancy, $s=k \ln w$, (b) the sum over $i$ may be replaced by $\int \rho(E) d E$, and (c) the number of vacancies is not a conserved quantity, $\mu=0$. Then Eq. (A6) becomes

$$
N=e^{s / k} \int \frac{\rho(E)}{e^{\beta E}+1} d E
$$

for vacancies as noninteracting fermions.

For vacancies as noninteracting bosons, one proceeds from Eq. (A4), noting that the occupation numbers are unrestricted, so that the sum is over all integers, to obtain

$$
Z=\prod_{i}\left[1-e^{-\beta\left(e_{i}-\mu\right)}\right]^{-1}
$$

From Eqs. (A2) and (A3) with the same simplifying assumptions as before one finally obtains

$$
N=e^{s / k} \int \frac{\rho(E)}{e^{\beta E}-1} d E
$$

for vacancies as noninteracting bosons. For other discussion, see Pushkarov. ${ }^{42}$

\section{ACKNOWLEDGMENT}

This work was supported in part by the U.S. Department of Energy, Division of Materials Sciences, under contract DOE-DE-AC01-76ER01198. One of us (P.R.G.) is pleased to acknowledge the support of fellowships from the General Electric Company and the Exxon USA Foundation.

\section{REFERENCES}

1. H. R. Glyde, in Rare Gas Solids, Vol. I, M. L. Klein and J. A. Venables, eds. (Academic Press, New York, 1975), Chapter 8.

2. R. O. Hilleke, P. Chaddah, R. O. Simmons, D. L. Price, and S. K. Sinha, Phys. Rev. Lett. 52, 847 (1984).

3. R. A. Guyer, R. C. Richardson, and L. I. Zane, Rev. Mod. Phys. 43, 532 (1971); S. B. Trickey, W. P. Kirk, and E. D. Adams, Rev. Mod. Phys. 44, 668 (1972); M. Roger, J. H. Hetherington, and J. M. Delrieu, Rev. Mod. Phys. 55, 1 (1983); M. C. Cross and D. S. Fisher, Rev. Mod. Phys. 57, 881 (1985).

4. R. Balzer and R. O. Simmons, in Low Temperature Physics-LT13, Vol. II, D. K. Timmerhaus, W. J. O'Sullivan, and E. F. Hammel, eds. (Plenum Press, New York, 1974), p. 115.

5. S. M. Heald, D. R. Baer, and R. O. Simmons, Solid State Commun. 47, 807 (1983).

6. S. M. Heald, D. R. Baer, and R. O. Simmons, Phys. Rev. B 30, 2531 (1984).

7. J. H. Hetherington, Phys. Rev. 176, 231 (1968).

8. R. A. Guyer, in Solid State Physics, Vol. 23, F. Seitz, D. Turnbull, and H. Ehrenreich, eds. (Academic Press, New York, 1969), p. 413. 
9. A. F. Andreev, in Progress in Low Temperature Physics, Vol. 8, D. F. Brewer, ed. (NorthHolland, New York, 1982), Chapter 2.

10. A. Landesmann, J. Low Temp. Phys. 30, 117 (1978).

11. S. E. Kal'noi and M. A. Strzhemechnyi, Fiz. Nizk. Temp. 8, 1025 (1982) [Sov. J. Low Temp. Phys. 8, 515 (1982)].

12. E. R. Grilly, J. Low Temp. Phys. 11, 33 (1973).

13. J. K. Hoffer, W. R. Gardner, C. G. Waterfield, and N. E. Phillips, J. Low Temp. Phys. 23, 63 (1976).

14. J. D. Eshelby, in Solid State Physics, Vol. 3, F. Seitz and D. Turnbull, eds. (Academic Press, New York, 1956), Chapter 3.

15. R. D. Fouchaux and R. O. Simmons, Phys. Rev. 136, A1664 (1964).

16. R. A. Guyer, J. Low Temp. Phys. 8, 427 (1972); H. A. Goldberg and R. A. Guyer, J. Low Temp. Phys. 28, 449 (1977).

17. W. F. Brinkman and T. M. Rice, Phys. Rev. B 2, 1324 (1970).

18. A. F. Andreev and I. M. Lifshitz, Zh. Eksp. Teor. Fiz. 56, 2057 (1969) [Sov. Phys,-JETP 29, 1107 (1969)].

19. M. E. Bernier and G. Guerrier, Physica 121B, 202 (1983); M. E. R. Bernier, J. Low Temp. Phys. 56, 205 (1984), and references therein.

20. M. Chapellier, M. Bassou, M. Devoret, J. M. Delrieu, and N. S. Sullivan, Phys. Rev. B 30, 2940 (1984).

21. N. E. Sullivan and P. Kumar, Phys. Rev. Lett. 55, 953 (1985).

22. W. P. Kirk, Bull. Am. Phys. Soc. 30, 775 (1984).

23. C. P. Flynn, Point Defects and Diffusion (Clarendon Press, Oxford, 1972), Chapters 2 and 7.

24. B. A. Fraass, P. R. Granfors, R. O. Hilleke, and R. O. Simmons, Rev. Sci. Instrum. 55, 1455 (1984).

25. S. M. Heald and R. O. Simmons, Rev. Sci. Instrum. 48, 316 (1977).

26. V. B. Efimov and L. P. Mezhov-Deglin, Fiz. Nizk. Temp. 2, 652 (1976) [Sov. J. Low Temp. Phys. 2, 322 (1976)].

27. E. R. Grilly and R. L. Mills, Ann. Phys. (N.Y.) 18, 250 (1962).

28. W. R. Gardner, J. K. Hoffer, and N. E. Phillips, Phys. Rev. A 7, 1029 (1972).

29. R. W. Hill and O. V. Lounasmaa, Phil. Trans. R. Soc. (Lond.) 252, 357 (1960).

30. V. N. Grigor'ev, B. N. Esel'son, and V. A. Mikheev, Zh. Eksp. Teor. Fiz. 66, 321 (1974) [Sov. Phys.-JETP 39, 153 (1974)]; Fiz. Nizk. Temp. 1, 5 (1975) [Sov. J. Low Temp. Phys. 1, $1(1975)]$.

31. D. S. Miyoshi, R. M. Cotts, A. S. Greenberg, and R. C. Richardson, Phys. Rev. A 2, 870 (1970).

32. A. R. Allen and M. G. Richards, Phys. Lett. 65A, 36 (1978); A. R. Allen, M. G. Richards, and J. Schratter, J. Low. Temp. Phys. 47, 289 (1982); J. Schratter, A. R. Allen, and M. G. Richards, J. Low. Temp. Phys. 57, 179 (1984).

33. A. H. Dahm, in Progress in Low Temperature Physics, Vol. 10, D. F. Brewer, ed. (NorthHolland, New York, 1985), Chapter 1.

34. A. I. Golov, Y. B. Efimov, and L. P. Mezhov-Deglin, Pis'ma Zh. Eksp. Teor. Fiz. 40, 293 (1984) [JETP Lett. 40, 1080 (1984)].

35. H. A. Reich, Phys. Rev. 129, 630 (1963).

36. N. Sullivan, G. Deville, and A. Landesman, Phys. Rev. B 11, 1858 (1975).

37. H. R. Glyde and F. C. Khanna, Can. J. Phys. 50, 1143 (1972).

38. D. S. Greywall, Phys. Rev. B 13, 1056 (1976).

39. D. O. Edwards and R. C. Pandorf, Phys. Rev. 140, A816 (1965).

40. J. Holder and A. V. Granato, Phys. Rev. 182, 729 (1969).

41. B. A. Fraass, P. R. Granfors, and R. O. Simmons, unpublished.

42. D. I. Pushkarov, Phys. Stat. Sol. (B) 133, 525 (1986). 